\title{
Human antibodies that neutralize respiratory droplet transmissible H5N1 influenza viruses
}

\author{
Natalie J. Thornburg, ${ }^{1}$ David P. Nannemann, ${ }^{2}$ David L. Blum, ${ }^{1}$ Jessica A. Belser, ${ }^{3}$ \\ Terrence M. Tumpey, ${ }^{3}$ Shyam Deshpande, ${ }^{1}$ Gloria A. Fritz, ${ }^{1}$ Gopal Sapparapu, ${ }^{1}$ Jens C. Krause, ${ }^{1,4}$ \\ Jeong Hyun Lee, ${ }^{5}$ Andrew B. Ward, ${ }^{5}$ David E. Lee, ${ }^{6}$ Sheng Li, ${ }^{6}$ Katie L. Winarski, ${ }^{7}$ \\ Benjamin W. Spiller, ${ }^{7,8}$ Jens Meiler, ${ }^{2,8}$ and James E. Crowe Jr.1,4,7
}

\begin{abstract}
${ }^{1}$ The Vanderbilt Vaccine Center and 2Department of Chemistry, Vanderbilt University, Nashville, Tennessee, USA. ${ }^{3}$ nfluenza Division, Centers for Disease Control and Prevention, Atlanta, Georgia, USA. ${ }^{4}$ Department of Pediatrics, Vanderbilt University, Nashville, Tennessee, USA. ${ }^{5}$ Department of Integrative Structural and Computational Biology, The Scripps Research Institute, La Jolla, California, USA. ${ }^{6}$ Department of Medicine and Biomedical Sciences Graduate Program, School of Medicine, UCSD, San Diego, California, USA. ${ }^{7}$ Department of Pathology, Microbiology and Immunology, and ${ }^{8}$ Department of Pharmacology, Vanderbilt University, Nashville, Tennessee, USA.
\end{abstract}

\begin{abstract}
Recent studies described the experimental adaptation of influenza H5 HAs that confers respiratory droplet transmission (rdt) to influenza virus in ferrets. Acquisition of the ability to transmit via aerosol may lead to the development of a highly pathogenic pandemic $\mathrm{H} 5$ virus. Vaccines are predicted to play an important role in H5N1 control should the virus become readily transmissible between humans. We obtained PBMCs from patients who received an A/Vietnam/1203/2004 H5N1 subunit vaccine. Human hybridomas were then generated and characterized. We identified antibodies that bound the HA head domain and recognized both WT and rdt H5 HAs. We used a combination of structural techniques to define a mechanism of antibody recognition of an $\mathrm{H} 5 \mathrm{HA}$ receptor-binding site that neutralized $\mathrm{H} 5 \mathrm{~N} 1$ influenza viruses and pseudoviruses carrying the HA rdt variants that have mutations near the receptor-binding site. Incorporation or retention of this critical antigenic site should be considered in the design of novel $\mathrm{H} 5 \mathrm{HA}$ immunogens to protect against mammalianadapted H5N1 mutants.
\end{abstract}

\section{Introduction}

There have been several outbreaks of highly pathogenic H5N1 avian influenza viruses affecting humans over the past decade, the causes of which have been limited to direct human contact with infected birds. Recent publications suggest that a very small number of coding mutations in the viral HA gene render the virus transmissible via respiratory droplets between ferrets $(1,2)$. Humans lack potent immunity against influenza viruses carrying the H5 HA, and therefore the natural occurrence of a mammalianadapted high pathogenicity H5N1 influenza virus might cause a major pandemic marked by high mortality. A number of experimental H5N1 influenza vaccines have been tested in clinical trials. In the recent publications of the ferret adaptation studies, investigators determined that plasma from individuals vaccinated with conventional $\mathrm{H} 5 \mathrm{~N} 1$ vaccines had neutralizing activity against $\mathrm{rdt}$ viruses $(1,2)$. It is poorly understood how antibodies against vaccines could bind and neutralize both WT and rdt H5N1 viruses. The public release of the sequences of the associated HAs $(1,2)$ allowed us to determine rapidly the molecular mechanism for how conventional H5 vaccines provide protection against these viruses and how they may provide protection against likely naturally occurring human-adapted $\mathrm{H} 5 \mathrm{~N} 1$ variant viruses in the future.

\section{Results and Discussion}

We generated human hybridomas and cloned antibodies from PBMCs from 4 subjects vaccinated against a monovalent inactivated subvirion vaccine incorporating the HA from A/Vietnam/1203/2004 (VN/1203) H5N1 influenza virus (Supplemental

Conflict of interest: The authors have declared that no conflict of interest exists. Citation for this article: J Clin Invest. 2013;123(10):4405-4409. doi:10.1172/JCI69377.
Table 1; supplemental material available online with this article; doi:10.1172/JCI69377DS1). The antibodies included diverse clones encoded by $\mathrm{V}_{\mathrm{H}}$ gene families 1 , 3, or 4 and had HCDR3 loops varying in length from 15 to 22 amino acids (Supplemental Table 1). The phenotype of antibodies in hemagglutination inhibition (HI) and neutralization assays (Table 1) indicated the panel included clones that bound HA head domains as well as one that putatively bound to the stem domain. Antibodies that were weakly neutralizing $\left(\mathrm{IC}_{50}, 5 \mu \mathrm{g} / \mathrm{ml}\right)$ were not confirmed to be neutralizing by independent testing at a second site in our collaborative group, and therefore we did not delineate them as stem or head binding. Potent HI activity indicated specificity for the HA head domain. Several of the head domain-binding antibodies exhibited potent antiviral activity, with neutralizing capacity in the nM range (Table 1). In addition to the 3 mAbs H5.16, H5.22, and H5.24 that intermittently showed weak neutralizing capability, we also isolated a number of additional antibodies that bound to H5 HA but did not appear to neutralize (data not shown).

H5N1 field strains exhibit genetic and antigenic diversity in HA. We tested the breadth of these antibodies against field strains by determining the half-maximal effective concentration $\left(\mathrm{EC}_{50}\right)$ value of binding to HAs from 7 H5N1 field strains. Potently neutralizing antibodies H5.2 and H5.9 bound all strains of H5 influenza HA tested (Supplemental Table 2). Potently neutralizing antibodies H5.3 and H5.13 recognized a more restricted set of H5 HAs (Supplemental Table 2). All H5 influenza field strain HAs we tested have either a K or an R at position 193, which is located on the rim of the receptor-binding pocket (Supplemental Figure 1 and refs. 3 , 4). We constructed point mutant variants, K193R and K193S VN/1203 HAs. MAb H5.3 did not bind the VN/1203 HA K193R variant, and MAbs H5.3 and H5.2 did not bind the VN/1203 HA 
Table 1

H5-specific human mAbs: functional activity

\begin{tabular}{|c|c|c|c|c|c|c|c|c|c|c|}
\hline \multirow[t]{2}{*}{ Subject } & \multirow[t]{2}{*}{$\mathrm{mAb}$} & \multirow{2}{*}{$\begin{array}{c}\text { HAI } \\
(\mu \mathrm{g} / \mathrm{ml}) \\
\text { VN/1203 }\end{array}$} & \multicolumn{2}{|c|}{$\begin{array}{l}\text { Neutralization, IC } C_{50} \\
(\mu \mathrm{g} / \mathrm{ml})\end{array}$} & \multirow[t]{2}{*}{$\begin{array}{l}\text { Head } \\
\text { vs. stem }\end{array}$} & \multicolumn{5}{|c|}{ Pseudovirion neutralization, $\mathrm{IC}_{50}(\mu \mathrm{g} / \mathrm{ml})$} \\
\hline & & & VN/1203 & WSN/33 & & WT VN/1203 & rdt VN/1203 K & VN/1203 F & WT Indo/05 & rdt Indo/05 F \\
\hline 18 & $\begin{array}{l}\mathrm{H} 5.2 \\
\mathrm{H} 5.3 \\
\mathrm{H} 5.7 \\
\mathrm{H} 5.9\end{array}$ & $\begin{array}{c}0.08 \\
0.08 \\
> \\
0.08\end{array}$ & $\begin{array}{c}0.07 \\
0.02 \\
5 \\
0.04\end{array}$ & $\begin{array}{c}5 \\
> \\
> \\
0.16\end{array}$ & $\begin{array}{l}\text { HeadA }^{A} \\
\text { Head } \\
\text { Stem } \\
\text { Head }\end{array}$ & $\begin{array}{c}0.005 \\
0.007 \\
0.17 \\
0.012\end{array}$ & $\begin{array}{c}0.005 \\
0.018 \\
0.19 \\
0.016\end{array}$ & $\begin{array}{c}0.005 \\
0.005 \\
0.07 \\
0.002\end{array}$ & $\begin{array}{c}>^{\mathrm{B}} \\
> \\
0.03 \\
0.47\end{array}$ & $\begin{array}{c}> \\
> \\
0.11 \\
>D\end{array}$ \\
\hline 29 & $\begin{array}{l}\mathrm{H} 5.13 \\
\mathrm{H} 5.16\end{array}$ & $\begin{array}{c}0.08 \\
>\end{array}$ & $\underset{>E}{0.04}$ & $\begin{array}{l}> \\
>\end{array}$ & $\begin{array}{l}\text { Head } \\
\text { NT }\end{array}$ & $\begin{array}{c}0.04 \\
\text { NT }\end{array}$ & $\begin{array}{c}0.02 \\
\text { NT }\end{array}$ & $\begin{array}{l}0.03 \\
\text { NT }\end{array}$ & $\stackrel{>}{\text { NT }}$ & $\stackrel{>}{\text { NT }}$ \\
\hline 42 & $\begin{array}{l}\text { H5.22 } \\
\text { H5.24 } \\
\text { H5.31 }\end{array}$ & $\begin{array}{c}> \\
> \\
1.3\end{array}$ & $\begin{array}{l}>^{E} \\
>^{E} \\
0.2\end{array}$ & $\begin{array}{l}> \\
> \\
>\end{array}$ & $\begin{array}{l}\text { NT } \\
\text { NT } \\
\text { Head }\end{array}$ & $\begin{array}{l}\text { NT } \\
\text { NT } \\
2.9\end{array}$ & $\begin{array}{c}\text { NT } \\
\text { NT } \\
0.55^{\mathrm{D}}\end{array}$ & $\begin{array}{c}\text { NT } \\
\text { NT } \\
0.07^{D}\end{array}$ & $\begin{array}{l}\text { NT } \\
\text { NT } \\
>\end{array}$ & $\begin{array}{l}\text { NT } \\
\text { NT } \\
>\end{array}$ \\
\hline 54 & H5.36 & $>$ & 2.5 & $>$ & ND & 2.5 & $0.16^{\mathrm{D}}$ & $0.005^{\mathrm{D}}$ & 0.10 & 0.15 \\
\hline
\end{tabular}

A Head domain binding phenotype was assigned when the mAb possessed both $\mathrm{HI}$ and neutralizing activity. ${ }^{\mathrm{B}}>$ indicates activity was not detected at any concentration tested, up to $5 \mu \mathrm{g} / \mathrm{ml}$. CPutative stem domain binding phenotype was assigned when the mAb possessed neutralizing but not $\mathrm{HI}_{\text {activity. }} \mathrm{DIC}_{50}$ differs from parental strain, as determined by nonoverlapping $95 \% \mathrm{Cls}$. Eln some neutralization assays, a low level of neutralizing activity was detected at $5 \mu \mathrm{g} / \mathrm{ml}$, but this finding could not be reproduced reliably. WSN/33, A/WSN/1933 (H1N1); NT, binding domain not tested because of absence of neutralizing activity; ND, domain not determined because of complex results in the functional mapping experiments.

K193S variant (Supplemental Table 2). These data indicated these antibodies recognize and block the receptor-binding site on HA with an epitope that involves the 190 loop.

Two recent publications suggest that coding mutations in the head domain of the viral H5 HA gene render the virus rdt between ferrets (refs. 1, 2; Supplemental Figure 1). The numbering used in the 2 papers on derivation of rdt viruses differed, as one used $\mathrm{H} 3$ numbering (1) and the other used absolute position (2). In this manuscript, we use $\mathrm{H} 3$ numbering. The surface changes within the head domain reported were N158D/N224K/Q226L (in VN/1203) and T160A/Q226L/G228S (in A/Indonesia/05/2005 [Indo/05]). In order to determine whether our H5 influenza-neutralizing antibodies could bind the rdt H5N1 viruses, we engineered N158D/N224K/
Q226L from Kawaoka (K) laboratory strains into the VN/1203 HA (VN/1203 K) and T160A/Q226L/G228S from Fouchier laboratory strains (F) into both VN/1203 (VN/1203 F) and Indo/05 HA (Indo/05 F) variants and tested binding. The potently neutralizing antibodies retained the ability to bind the VN/1203 F and K variants with $\mathrm{EC}_{50}$ values similar to those of the WT H5 HAs (Supplemental Table 2). The same was true for the Indo/05 variant, with the exceptions of H5.2, H5.9, and H5.31. Antibody H5.2 exhibited a decrease in Indo/05 binding with the Indo/05 F rdt mutations, as determined by nonoverlapping 95\% CIs. Antibody H5.9 lost the ability to bind Indo/05 with the Indo/05 F rdt mutations. In contrast, antibody H5.31 gained the ability to bind the Indo/05 F rdt mutant, whereas it did not bind the WT Indo/05 HA (Supplemental Table 2).

A
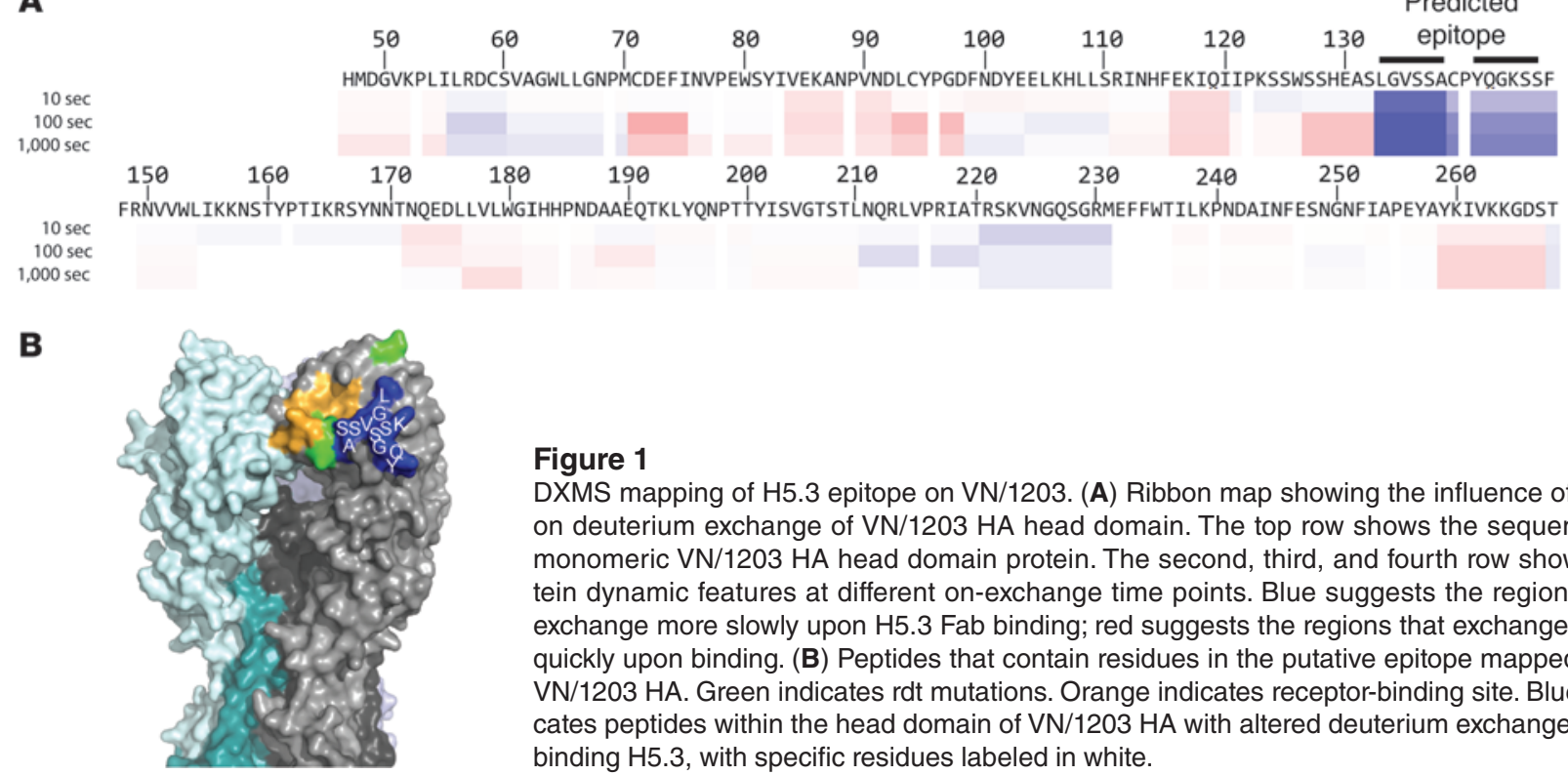

Figure 1

DXMS mapping of H5.3 epitope on VN/1203. (A) Ribbon map showing the influence of H5.3 on deuterium exchange of $\mathrm{VN} / 1203 \mathrm{HA}$ head domain. The top row shows the sequence of monomeric VN/1203 HA head domain protein. The second, third, and fourth row show protein dynamic features at different on-exchange time points. Blue suggests the regions that exchange more slowly upon H5.3 Fab binding; red suggests the regions that exchange more quickly upon binding. (B) Peptides that contain residues in the putative epitope mapped onto VN/1203 HA. Green indicates rdt mutations. Orange indicates receptor-binding site. Blue indicates peptides within the head domain of $\mathrm{VN} / 1203 \mathrm{HA}$ with altered deuterium exchange upon binding $\mathrm{H} 5.3$, with specific residues labeled in white. 


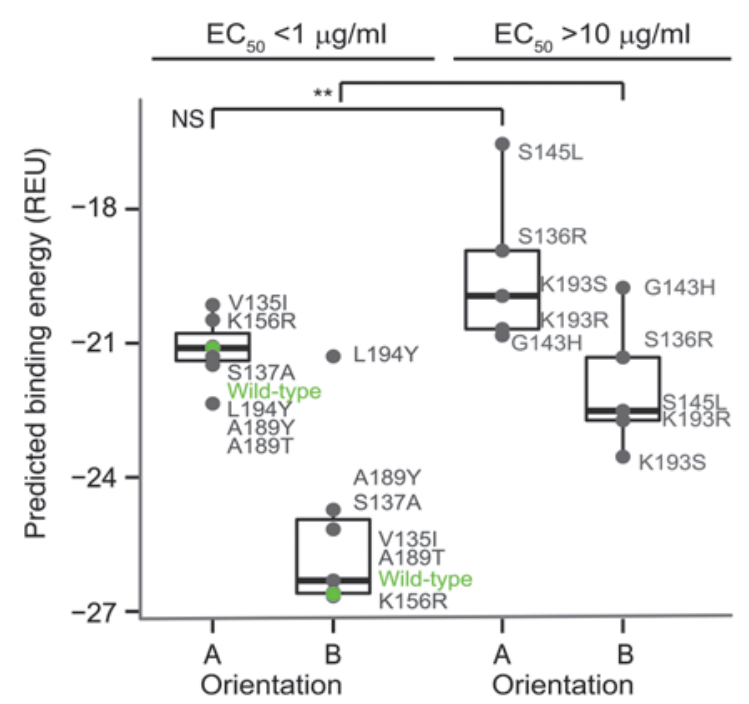

To better describe the molecular interactions that maintained binding of rdt variant HAs by mAb H5.3, we determined the structure of H5.3 by x-ray crystallography, refined the model to $2.25 \AA$ (Supplemental Table 3), and determined a low-resolution structure of H5.3 complexed with VN/1203 HA trimer by EM (Supplemental Figure 2). Using monomeric VN/1203 HA head domain, we also identified portions of the H5.3 epitope on HA by deuterium exchange mass spectrometry (DXMS), which indicated binding of the Fab to 2 peptides that encompass residues 130-144 (Figure 1). The crystal structures of trimeric VN/1203 HA and H5.3 were fit independently into the $16 \AA$ resolution EM density map of the complex. Two possible orientations of H5.3, rotated approximately $180^{\circ}$ to each other, were differentiated by cross-correlation coefficients of 0.895 and 0.868 (Supplemental Figure 3).

We used these structural data to establish an atomic detail model of the H5.3 interface with VN/1203 HA. H5.3 was docked

\section{Figure 2}

Experimentally determined binding affinities compared with in silicopredicted binding energies of VN/1203 HA variants for each EM density fitting orientation. Tighter binding is indicated by a more negative binding energy ( $y$ axis). The mutants were divided into binder, those with $\mathrm{EC}_{50}$ less than $1 \mu \mathrm{g} / \mathrm{ml}$, and nonbinders, those with $\mathrm{EC}_{50}$ values greater than $10 \mu \mathrm{g} / \mathrm{ml}$. The predicted binding energies of the binders did not differ significantly from nonbinders in orientation A (NS). The predicted binding energies of the binders did differ significantly from the nonbinders in orientation $\mathrm{B} .{ }^{* \star} P$ value between 0.001 and 0.1 . WT or native VN/1203 binding is indicated in green. The bounds of the boxes indicate the 75th (top) and 25th (bottom) percentiles; the central lines within the boxes indicate the average value; the whiskers extend to the furthest value that extends 1.5 times the distance between the first and third quartiles; data points outside the whiskers indicate outlying values beyond the $\times 1.5$ distance.

A
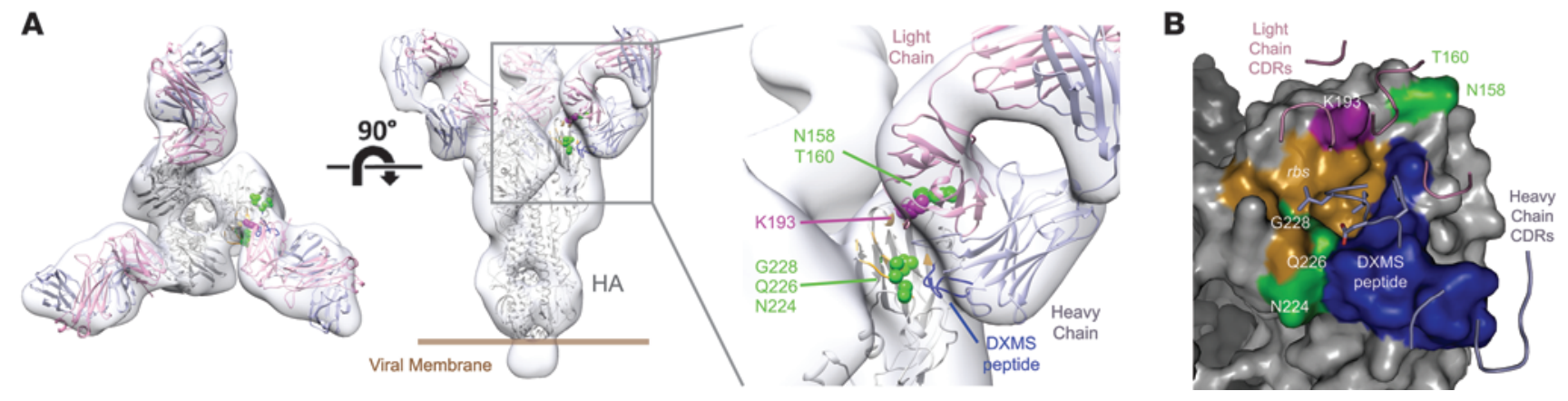

\section{Figure 3}

Computational modeling of mAb H5.3 complex with VN/1203 HA. (A) Model of H5.3 in complex with VN/1203 HA overlaid with EM density map in transparent pale blue. VN/1203 HA is shown as a gray ribbon with heavy chain in light blue and light chain in pink. Green spheres show the position of variant mutations in the rdt VN/1203 or Indonesia strains. K193 is shown as magenta spheres. The peptide containing contact residues identified by DXMS is indicated in dark blue, and residues in the receptor-binding site are indicated in orange. The image to the right shows the preferred binding mode with light chain on top (orientation B). (B) Interaction of H5.3 complementarity determining region with VN/1203 HA head domain. The surface of HA is represented with residues of the receptor-binding site colored bright orange, variant mutations from the rdt VN/1203 or Indonesia strains colored green, and residues in the peptide with altered deuterium exchange upon H5.3 binding colored dark blue. Heavychain complementarity determining regions (CDRs) are in shades of blue; light-chain CDRs and framework region 3 are in shades of pink. The K193 residue, colored in magenta, interacts with a polar cavity formed by the light chain. Residues 110-111.2 of HCDR3 (IMGT numbering, shown as sticks) insert into the receptor-binding site. The H5.3 footprint avoids significant contact with rdt mutant residues. 
B also was confirmed by docking to the recently published crystal structure of the HA from the rdt VN/1203 K strain (11).

The structure of the complex revealed H5.3 insertion of HCDR3 into the HA receptor-binding site (Figure 3B). This mechanism is a recurring motif in antibody binding of the influenza HA head domain (7-13). The wide footprint of mAb H5.3 provides for excellent binding affinity but prevents broad neutralizing capacity. Comparison of H5.3 to cocrystal structures of previously described antibodies that insert HCDR3 into the receptor-binding site indicated a distinct approach angle for the H5.3 Fab (Supplemental Figure 4). This angle of approach, which spans the region between the rdt mutations, makes mAb H5.3 resistant to escape from the specific mutations present in the rdt viral variants.

There is currently a hold on research using viral variants containing rdt mutations, and therefore these antibodies cannot be tested for their neutralizing activities against live rdt viruses. We produced lentivirus-based pseudovirions packaged with WT VN/1203 HA, VN/1203 K variant, VN/1203 F variant, WT Indo/05 $\mathrm{HA}$, or Indo/05 F variant HA. We tested antibody neutralization of the pseudovirions (Table 1). Neutralizing antibodies neutralized pseudovirions packaged with WT or Vietnam-based mutant HAs at similar concentrations, with the exceptions of H5.31 and H5.36, which neutralized pseudovirions packaged with mutant HAs more potently than WT HA, as determined by nonoverlapping 95\% CIs. Due to conflicting data from competition, neutralization, HI, and pseudovirus neutralization assays, we were unable to determine whether H5.36 binds to head or stem. As expected, antibodies that did not bind Indo/05 HA also did not neutralize Indonesia-based pseudoviruses (Table 1). All of the antibodies, except H5.9, neutralized WT and variant Indonesia-based pseudoviruses at similar $\mathrm{IC}_{50}$ values (Table 1). Consistent with the binding data, antibody H5.9 lost the ability to neutralize the Indo/05 F variant.

This study confirms at the clonal level previously published studies with polyclonal sera suggesting that conventional subunit vaccine regimens directed against highly pathogenic $\mathrm{H} 5 \mathrm{~N} 1$ avian influenza viruses elicit human B cell responses encoding potently neutralizing antibody clones that are able to bind $\mathrm{rdt}$ viral variants $(1,4)$. Furthermore, the structural and computational studies of H5.3 presented in this paper suggest a molecular mechanism for how conventional vaccines can elicit mAbs that exhibit $\mathrm{HI}$ activity and potently neutralize conventional and $\mathrm{rdt} \mathrm{H} 5 \mathrm{~N} 1$ variants, even though the epitope and the rdt mutations are both located in the receptor-binding site. The current pause in rdt avian influenza live virus research prevents us from testing the neutralizing or protective capacity against virus infection. However, the fact that the antibodies neutralize the parental strains of virus, neutralize pseudovirions with the rdt HA molecules, and bind to the WT and rdt variant HAs with comparable $\mathrm{EC}_{50}$ values suggests that these $\mathrm{mAbs}$ also will neutralize the rdt viruses with high potency. It is encouraging that the binding of several of the most potently neutralizing antibodies that bind in or near the receptor-binding site is not affected by the variations conferring respiratory droplet transmissibility, even though the mutations are located in a similar region.

\section{Methods}

Statistics. In neutralization assays, $\mathrm{IC}_{50}$ values were calculated after log transformation of antibody concentrations using a 3-parameter nonlinear fit analysis of antibody $\log _{10}$ concentration versus response with $R^{2}$ values greater than 0.85 . IC S0 $_{50}$ values with nonoverlapping $95 \%$ CIs were identified as differing significantly. In ELISA assays, EC $_{50}$ values were calculated after log transformation of antibody concentrations using sigmoidal doseresponse nonlinear fit analysis with $R^{2}$ values greater than 0.85 . The $\mathrm{EC}_{50}$ values that retained nonoverlapping $95 \%$ CIs were identified as differing significantly. To analyze ELISA data obtained for testing complex orientation, a 1-way ANOVA statistical analysis was performed to compare the sets of variants with $\mathrm{EC}_{50}$ values of less than $1 \mu \mathrm{g} / \mathrm{ml}$ to variants with no detectable binding at $10 \mu \mathrm{g} / \mathrm{ml}$. All statistics were analyzed using Prism software version 5 (GraphPad).

Study approval. PBMCs were collected in the Vanderbilt Clinical Trials Center after informed consent from otherwise healthy subjects with prior history of experimental H5N1 subunit vaccination, as described in Supplemental Methods. The protocol and consent form were approved prior to study by the Vanderbilt University Institutional Review Board Committee.

Deposits. Antibody nucleotide sequences have been deposited in GenBank (JX4589933-52); antibody X-ray structure has been deposited in the Protein Data Bank (4GSD).

\section{Acknowledgments}

The authors thank P. McGraw, R. Falk, and F. Smith-House for technical support, J.A. Archuleta, C. Kinnard, and J. Janssen of the Vanderbilt Clinical Trials Center for clinical support, and S.A. Smith for helpful discussions. The authors acknowledge the significant contributions of Virgil Woods, who is now deceased. The original clinical trial of the H5 vaccine (DMID 04-062) was supported by NIH and performed in the Vanderbilt Vaccine and Treatment Evaluation Unit (PI: K.M. Edwards). This work was supported by NIH grant R01 AI106002, NIAID contract HHSN272200900047C, the Vanderbilt NIH CTSA grant UL1 RR024975, and the DoD grant HDTRA1-10-1-0067. Use of the Advanced Photon Source was supported by the US Department of Energy, Office of Science, Office of Basic Energy Sciences, under contract No. DE-AC02-06CH11357. K.L. Winarski was supported by a Molecular Biophysics training grant at Vanderbilt (NIH T32 GM008320). Use of the LS-CAT sector 21 was supported by the Michigan Economic Development Corp. and the Michigan Technology Tri-Corridor for the support of this research program (Grant 085P1000817). The electron microscopy studies were supported by startup funds from The Scripps Research Institute (to A.B. Ward) and conducted at the National Resource for Automated Molecular Microscopy, which is supported by NIH through the NCRR P41 program (RR017573). The modeling studies were conducted using the resources of the Advanced Computing Center for Research and Education at Vanderbilt. The funders had no role in study design, data collection and analysis, decision to publish, or preparation of the manuscript.

Received for publication February 20, 2013, and accepted in revised form July 3, 2013.

Address correspondence to: James E. Crowe Jr., Vanderbilt Vaccine Center, Vanderbilt University Medical Center, 11475 MRB IV, 2213 Garland Avenue, Nashville, Tennessee 37232-0417, USA. Phone: 615.343.8064; Fax: 615.343.4456; E-mail: james.crowe@ vanderbilt.edu.
Nature. 2012;486(7403):420-428.

2. Herfst $S$, et al. Airborne transmission of influenza A/H5N1 virus between ferrets. Science.
2012;336(6088):1534-1541.

3. Yu X, et al. Neutralizing antibodies derived from the B cells of 1918 influenza pandemic survivors. 
Nature. 2008;455(7212):532-536.

4. Bernstein DI, et al. Effects of adjuvants on the safety and immunogenicity of an avian influenza H5N1 vaccine in adults. Infect Dis. 2008;197(5):667-675.

5. Chaudhury S, et al. Benchmarking and analysis of protein docking performance in Rosetta v3.2. PLoS One. 2011;6(8):e22477.

6. Dimaio F, Tyka MD, Baker ML, Chiu W, Baker D. Refinement of protein structures into lowresolution density maps using rosetta. J Mol Biol. 2009;392(1):181-190.

7. Tsibane $\mathrm{T}$, et al. Influenza human monoclonal antibody $1 \mathrm{~F} 1$ interacts with three major antigenic sites and residues mediating human receptor specificity in H1N1 viruses. PLoS Pathog. 2012; 8(12):e1003067.

8. Whittle JRR, et al. Broadly neutralizing human antibody that recognizes the receptor-binding pocket of influenza virus hemagglutinin. Proc Nat Acad Sci U S A. 2011;108(34):14216-14221.

9. Bizebard T, et al. Structure of influenza virus haemagglutinin complexed with a neutralizing antibody. Nature. 1995;376(6535):92-94.

10. Barbey-Martin C, et al. An antibody that prevents the hemagglutinin low $\mathrm{pH}$ fusogenic transition. Virology. 2002;294(1):70-74.

11. Ekiert DC, et al. Cross-neutralization of influenza A viruses mediated by a single antibody loop. Nature. 2012;489(7417):526-532.

12. Schmidt AG, et al. Preconfiguration of the antigenbinding site during affinity maturation of a broadly neutralizing influenza virus antibody. Proc Natl Acad Sci U S A. 2013;110(1):264-269.

13. Xu RR, et al. A recurring motif for antibody recognition of the receptor-binding site of influenza hemagglutinin. Nat Struct Mol Biol. 2013;20(3):363-370. 